\title{
Neutron-Nucleus Total Elastic Scattering
}

\author{
Cvavb Chandra Raju \\ Department of Physics, Osmania University, Hyderabad, India \\ Email: cvavbc@gmail.com
}

Received 11 December 2014; accepted 24 December 2014; published 14 January 2015

Copyright (C) 2015 by author and Scientific Research Publishing Inc.

This work is licensed under the Creative Commons Attribution International License (CC BY).

http://creativecommons.org/licenses/by/4.0/

(c) (i) Open Access

\begin{abstract}
Using an exact and complete Yukawa Potential Energy (YPE), the neutron-triton and neutron-helium elastic total scattering cross section is estimated and extrapolated to zero energy. The estimated value agrees pretty well with the experimental value and the procedure can be extended to any neutron-nucleus total scattering. The results are extended to the case of ${ }^{4} \mathrm{He},{ }^{7} \mathrm{Li},{ }^{9} \mathrm{Be}$ and ${ }^{27} \mathrm{Al}$ nuclei also.
\end{abstract}

\section{Keywords}

Totalelastic Scattering Cross-Section, Complete Yukawa Potential Energy, Born Approximation, One Pion Exchange Potential

\section{Introduction}

The theoretical description of $\mathrm{A}=4$ systems still constitutes a challenging problem from the stand-point of nuclear few body theory. Only recently the study of alpha particle bound state reached a satisfactory level of accuracy because of new numerical methods. The $4 \mathrm{~N}$ bound state has been calculated to a few tenths of $\mathrm{KeV}$ [1]-[3]. But these numerical methods are very involved. The total elastic scattering cross section $\sigma_{T}$ of a neutron by a Triton nucleus is discussed and compared to the experiment in [1]. Here we use a mass dependent Yukawa Potential Energy to estimate the total elastic scattering cross section of a neutron by a nucleus.

Instead of the $\mathrm{P}-{ }^{3} \mathrm{He}$ elastic scattering at low energies, the situation is simpler for the $\mathrm{n}-{ }^{3} \mathrm{H}$ elastic scattering. There is no Coulomb complication with the latter. Experimentally, only the total cross section $\sigma_{T}$ has been measured with high precision for large range of energies. The extrapolation of the measured $\sigma_{T}$ to zero energy is straightforward. The value obtained is [1],

$$
\sigma_{T}(0)=1.70 \pm 0.03 \mathrm{~b} .
$$

The above value is a very precisely measured value for the neutron-triton elastic scattering. There is another parameter called the coherent scattering length $r_{c}$. The coherent scattering length has been measured by neu- 
tron-interferometry techniques. The most recent value reported in the literature is [1],

$$
r_{c}=3.59 \pm 0.02 \mathrm{fm} \text {. }
$$

Estimation is also obtained from $\mathrm{P}^{3}{ }^{3} \mathrm{He}$ scattering data for the coherent scattering length through Coulombcorrected R-matrix theory. This gives [1],

$$
r_{c}=3.607 \pm 0.017 \mathrm{fm} \text {. }
$$

The above value is also an approximate experimental value. The theoretically obtained values are [1]-[3],

$$
\begin{aligned}
& \sigma_{T}(0)=1.73 \mathrm{~b}(\text { theoretical }), \\
& r_{c}=3.71 \mathrm{fm} \text { (theoretical). }
\end{aligned}
$$

In this paper we estimate the above experimental values with an exact Yukawa Potential Energy function and by using the methods of ordinary quantum mechanics. In Section 2, we present the relevant theory and apply it to the nucleus triton. In Section 3 the estimations are extended to many more nuclei with ease. We present our conclusions in Section 4.

\section{Complete Yukawa Potential Energy}

The complete Yukawa Potential Energy function operative for any nuclear process is given by,

$$
V(r)=-\frac{g^{2} \hbar c}{M_{0}^{2}} \frac{m_{1} M}{r} \mathrm{e}^{-a r},
$$

where, $m_{1}$ and $M$ are the masses of the interacting parts of the nuclear matter. For example for the neutronnucleus scattering $m_{1}$ is the rest mass of the neutron and $M$ is the rest mass of the scattering nucleus. There are two unknown parameters in the above YPE. The parameter $M_{0}^{2}$ is specific to each nucleus [4]-[7]. We have shown in [4]-[7] whenever the Yukawa factor $\mathrm{e}^{-a r}$ is set equal to one the resulting central potential yields the energy spectrum of the nucleus. By comparing the ground state energy with the known binding energy of the nucleus we can determine the parameter $M_{0}^{2}$. In our computations we will use this data from the references [4]-[7].

The other unknown is $a$ of the Yukawa factor. For One Pion Exchange Potential (OPEP),

$$
a=a_{1}=\frac{m_{\pi \pm} c}{\hbar}=6.930125 \times 10^{12} \frac{1}{\mathrm{~cm}},
$$

and,

$$
a=a_{2}=\frac{m_{\pi 0} c}{\hbar}=6.83996 \times 10^{12} \frac{1}{\mathrm{~cm}} .
$$

The parameter $g^{2}$ is same for all nuclei. It is given by,

$$
g^{2}=\frac{e^{2}}{0.2254}=\left(\frac{1}{137}\right) \frac{1}{0.2254}=0.032384
$$

We now have the entire YPE for any nucleus. For example for the nucleus ${ }^{3} \mathrm{H}$, the parameter $M_{0}^{2}$ is given by [4]-[6],

$$
M_{0}^{2}=1.103243 \times 10^{-48} \mathrm{~g}^{2} .
$$

We can now calculate the scattering amplitude for the scattering of a neutron of mass $m_{n}$ by a triton nucleus. In the first Born approximation, the scattering amplitude is given by,

$$
f(\vartheta)=\frac{2 \mu}{\hbar^{2}} \frac{g^{2} \hbar c m_{n} M}{M_{0}^{2}} \frac{1}{\left[a^{2}+2 k^{2}(1-\cos \vartheta)\right]},
$$

where, the reduced mass of the neutron is given by,

$$
\mu=\frac{m_{n} M}{m_{n}+M}
$$


Here, the wave number, $k^{2}=\frac{2 \mu E}{\hbar^{2}}, E$ being the CM energy of the incident neutron. The differential elastic scattering cross section can be obtained by squaring Equation (2.6). The total elastic scattering cross-section can then be obtained by integrating the differential cross-section. The Yukawa potential has the advantage relative to the Coulomb potential that certain integrals converge to finite numbers. The total elastic scattering-cross section is given by,

$$
\sigma_{T}(E)=\left[\frac{2 \mu}{\hbar^{2}} \frac{g^{2} \hbar c m_{n} M}{M_{0}^{2}}\right]^{2} \frac{4 \pi}{\left[a^{4}+4 a^{2} k^{2}\right]}
$$

The above expression gives the total elastic scattering-cross section for a neutron by any nucleus. It can be used for any neutron-nucleus scattering provided there are no resonances and there is no absorption. The total elastic scattering cross-section extrapolated to zero energy is given by,

$$
\sigma_{T}(0)=4 \pi r_{c}^{2},
$$

where,

$$
r_{c}=\frac{2 \mu}{\hbar^{2}} \frac{g^{2} \hbar c m_{n} M}{M_{0}^{2}} \frac{1}{a^{2}}
$$

The above Equations (2.9) and (2.10) can be used to compute the total scattering cross section at zero energy and the coherent scattering length. The required masses and other data are given in the table given in the next section. For ${ }^{3} \mathrm{H}$,

When $a=a_{1}$,

$$
\begin{aligned}
& \sigma_{T}(0)=1.6839 \text { barn } \\
& r_{1 c}=3.66059 \mathrm{fm} .
\end{aligned}
$$

And when, $a=a_{2}$,

$$
\begin{gathered}
\sigma_{T}(0)=1.7731 \text { barn } \\
r_{2 c}=3.75633 \mathrm{fm} .
\end{gathered}
$$

The above values obtained should be compared with the experimental value quoted in Equations (1.1) and (1.2). The average value of (2.11) and Equation (2.13) is 1.7285 barn. This should be compared with the value given by Equation (1.1). Similarly the average coherent radius is given by, $3.70846 \mathrm{fm}$.

\section{Theoretical Results for Some Nuclei}

Below we tabulate the estimated results for different nuclei. Table 1 is self-explanatory.

Table 1. The estimated results for different nuclei.

\begin{tabular}{ccccccccccc}
\hline Nucleus & Mass $\times 10^{-24} \mathrm{~g}$ & $M_{0}^{2} \times 10^{-48} \mathrm{~g}^{2}$ & $\begin{array}{c}\sigma_{T}(0) \text { in barn } \\
\text { when } a=a_{1}\end{array}$ & $\begin{array}{c}\sigma_{T}(0) \text { in barn } \\
\text { when } a=a_{2}\end{array}$ & $\begin{array}{c}\text { Average } \sigma_{T}(0) \\
\text { in barn }\end{array}$ & $\begin{array}{c}r_{c} \text { in fm } \\
a=a_{1}\end{array}$ & $\begin{array}{c}r_{c} \text { in fm } \\
a=a_{2}\end{array}$ & $\begin{array}{c}\text { Mean } \\
r_{c}\end{array}$ in fm \\
\hline${ }_{1}^{3} \mathrm{H}$ & 5.00830 & 1.103243 & 1.6839 & 1.7731 & 1.7285 & 3.661 & 3.76 & 3.71 \\
${ }_{2}^{3} \mathrm{He}$ & 5.00824 & 1.142021 & 1.57205 & 1.65499 & 1.61352 & 3.536 & 3.63 & 3.568 \\
${ }_{2}^{4} \mathrm{He}$ & 6.64648 & 0.935301 & 4.6875 & 4.9361 & 4.8118 & 6.11 & 6.27 & 6.19 \\
${ }_{3}^{7} \mathrm{Li}$ & 11.65036 & 0.872391 & 19.8359 & 20.8872 & 20.3615 & 12.56 & 12.9 & 12.73 \\
${ }_{4}^{9} \mathrm{Be}$ & 14.96509 & 0.973006 & 27.8388 & 29.3141 & 28.5765 & 14.88 & 15.3 & 15.09 \\
${ }_{13}^{27} \mathrm{Al}$ & 44.80393 & 1.094011 & 226.768 & 238.785 & 232.777 & 42.48 & 43.6 & 42.54 \\
\hline
\end{tabular}


For completeness, we have also included the estimation of the Triton nucleus as well. The ease with which the bulk nuclear properties can be obtained with the YPE can be seen from Table 1. The mass $M$ is obtained from Ref. [8].

The scattering of a neutron by Helium-3 or Helium-4 is quite difficult to measure. But scattering of neutrons of different energy by an Aluminum foil must not be very difficult to conduct. If measurements are made for different neutron energies for the elastic total scattering off Aluminum target our results can be put to test. This will pin down the non-relativistic YPE for any nucleus. Once it is confirmed, many bulk properties of a nucleus can be predicted with ease.

\section{Conclusion}

In this paper an exact YPE function is used to predict the total elastic scattering-cross section of a neutron by any nucleus. For all nuclei for which the parameter $M_{0}^{2}$ is known with certainty, the YPE is applied. The estimated results agree pretty well with the experimental values known for two nuclei, triton and helium-3. For other nuclei the experimental information is awaited. There are some nuclei like Aluminum for which the experiment can be carried out with ease. These tests would confirm the Yukawa potential. The nuclear "many-body" problem can readily be understood only by means of a correct potential energy function. The YPE function appears to be the right one in explaining the many gross properties of nuclei correctly. No sophiscated numerical procedures need be invoked to estimate the bulk properties of nuclei. Moreover the dependence of YPE on mass as in the case of gravitational interaction shows the beginnings of unifying the gravitational interaction with other interactions. The interaction constant $g^{2} \frac{m_{1} M}{M_{0}^{2}}$ is dimensionless in Equation (2.1) in units where $\hbar=c$ $=1$. Because of this the interaction is renormalizable.

\section{Acknowledgements}

The author is very grateful to V. G. Krishnan, Prudhvi R. Chintalapati and G. Prasad.

\section{References}

[1] Fisher, B.M., Brune, C.R., Karwowski, H.J., Leonard, D.S., Ludwig, E.J., et al. (2006) Physical Review C, C74, Article ID: 034001. http://dx.doi.org/10.1103/PhysRevC.74.034001

[2] Viviani, M., Girlanda, L., Kievsky, A. and Marcucci, L.E. (2013) Physical Review Letters, 111, Article ID: 172302. http://dx.doi.org/10.1103/PhysRevLett.111.172302

[3] Lazauskas, R. and Carbonell, J. (2011) Physical Review C, 84, Article ID: 034002. http://dx.doi.org/10.1103/PhysRevC.84.034002

[4] Raju, C.C. (2013) Journal of Nuclear and Particle physics, 3, 25-28.

[5] Raju, C.C. (2013) Journal of Modern Physics, 4, 459-462. http://dx.doi.org/10.4236/jmp.2013.44064

[6] Raju, C.C. (2013) Journal of Modern Physics, 4, 1180-1184.

[7] Raju, C.C. (2014) Modern Physics Letters A, 29, Article ID: 1450027. http://dx.doi.org/10.1142/S0217732314500278

[8] Wong, S.S.M. (1996) Introduction to Nuclear Physics. Prentice-Hall, 457. 
Scientific Research Publishing (SCIRP) is one of the largest Open Access journal publishers. It is currently publishing more than 200 open access, online, peer-reviewed journals covering a wide range of academic disciplines. SCIRP serves the worldwide academic communities and contributes to the progress and application of science with its publication.

Other selected journals from SCIRP are listed as below. Submit your manuscript to us via either submit@scirp.org or Online Submission Portal.
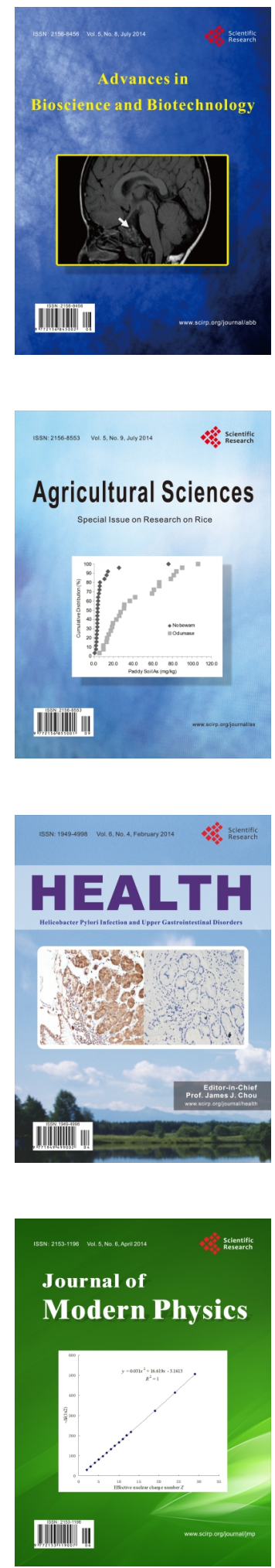
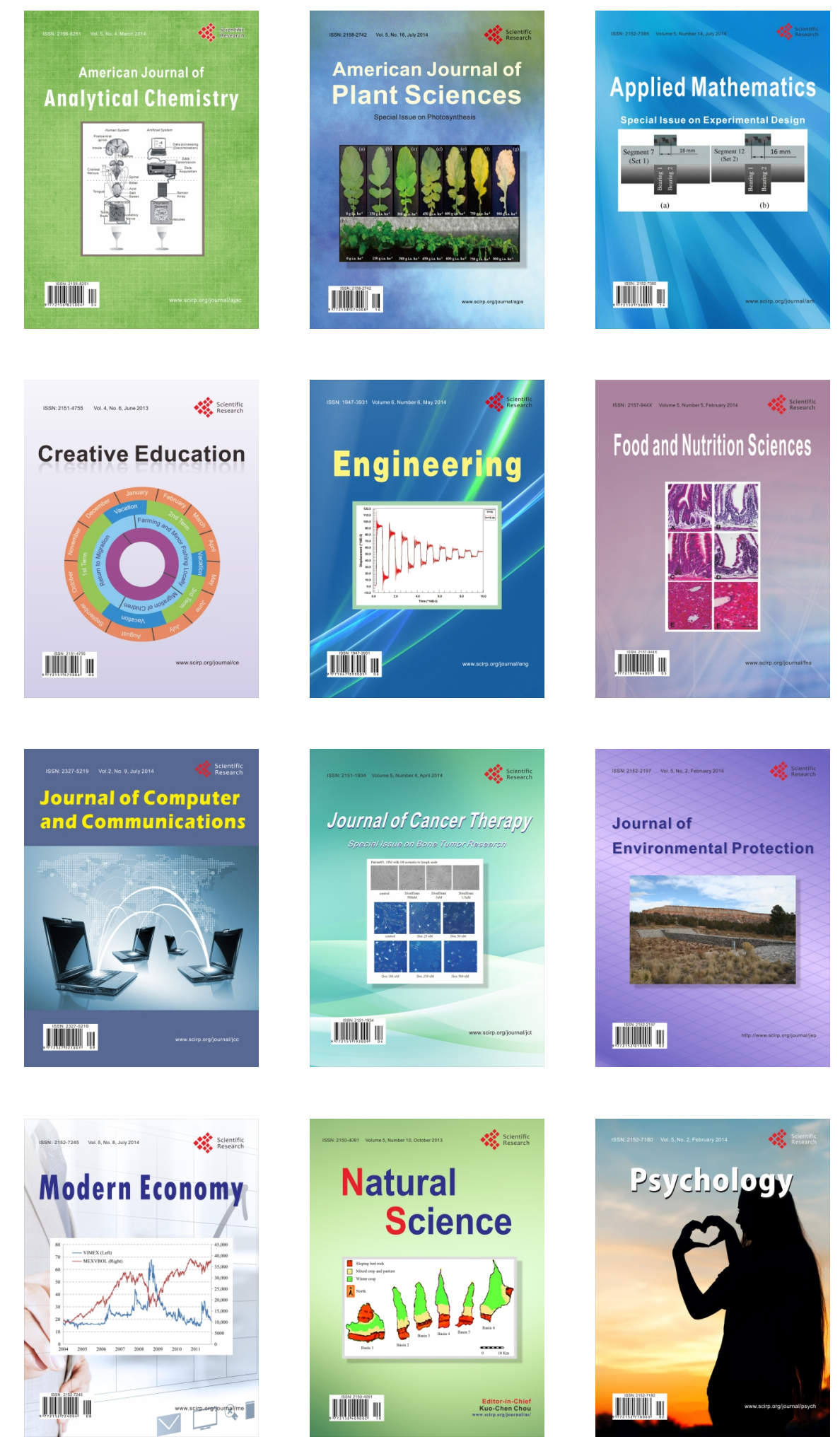